\title{
Trumpet Laminectomy Microdecompression for Lumbal Canal Stenosis
}

\author{
Jefri Henky ${ }^{1}$, Muneyoshi Yasuda ${ }^{2}$, Muhammad Zafrullah Arifin ${ }^{1}$, \\ Masakazu Takayasu ${ }^{2}$, Ahmad Faried $^{1}$ \\ ${ }^{1}$ Department of Neurosurgery, Faculty of Medicine, Universitas Padjadjaran-Dr. Hasan Sadikin General Hospital, Bandung, Indonesia \\ ${ }^{2}$ Department of Neurosurgery, Aichi Medical University Hospital, Nagakute, Aichi, Japan
}

Microsurgery techniques are useful innovations towards minimizing the insult of canal stenosis. Here, we describe the trumpet laminectomy microdecompression (TLM) technique, advantages and disadvantages. Sixty-two TLM patients with lumbar disc herniation, facet hypertrophy or yellow ligament or intracanal granulation tissue. The symptoms are low back pain, dysesthesia and severe pain on both legs. Spine levels operated Th11-S1; the patients who had trumpet-type fenestration, $62.9 \%$ had hypertrophy of the facet joint, $11.3 \%$ had intracanal granulation tissue, $79.1 \%$ had hypertrophy of the yellow ligament and $64.5 \%$ had disc herniation. The average of procedure duration was $68.9 \mathrm{~min}$ and intraoperative blood loss was $47.4 \mathrm{~mL}$. Intraoperative complications were found in $3.2 \%$ of patients, with dural damage but without cerebrospinal fluid leakage. The TLM can be performed for all ages and all levels of spinal canal stenosis, without the complication of spondilolistesis. The TLM has a shorter duration, with minimal intraoperative blood loss.

Keywords: Disc herniation; Facet joint hypertrophy; Intracanal granulation tissue; Lumbal canal stenosis; Trumpet laminectomy microdecompressionn

\section{Introduction}

Wide laminectomy via bilateral paraspinal exposure is the conventional surgical approach for the decompression of spinal canal stenosis. This classic technique allows maximal operative exposure for bilateral neural canal and/or foraminal decompression. There is a resulting extensive violation of the paraspinal muscles, the interspinous ligament, the supraspinous ligament, posterior bone elements and sometimes the capsular facet. Development of microsurgical techniques have provided innovations towards minimizing the surgical insult in surgical ap- proaches to canal stenosis [1].

The advantage of a microsurgical approach is the posibility of a wide bilateral decompression of spinal canal or foramen at one or multiple levels, through a minimal paraspinal muscular dissection. As a result, it is possible to preserve important soft tissues and bones, which are vital for the stability of the spinal column, while at the same time being able to remove bilateral pathologies encroaching upon the spinal canal or foramina [2].

Several authors have developed various microdecompression procedures for lumbar canal stenosis, including microhemilaminotomy, interlaminar microdecompres-

Received Nov 5, 2013; Revised Dec 19, 2013; Accepted Jan 24, 2014

Corresponding author: Jefri Henky

Department of Neurosurgery, Faculty of Medicine, Universitas Padjadjaran-Dr. Hasan Sadikin General Hospital,

Jl. Pasteur No. 38, Bandung 40161, West Java, Indonesia

Tel: +62-22-2041694, Fax: +62-22-2041694, E-mail: neurosurg_henky@yahoo.co.id 
sion, intersegmental microdecompression, resculpturing microlaminoplasty and segmental microsublaminoplasty [3]. In the Japanese Neurosurgical Society, one of the common procedures for microdecompression of lumbar spinal canal is trumpet laminectomy fenestration.

Trumpet laminectomy microdecompression is a trumpet fenestration, with splitting of the spinous process and partial laminectomy of the caudal portion of the laminae. It involves exposure of the laminae by longitudinally splitting the spinous process into two halves and cutting the base of the spinous process transversely, leaving its muscular and ligamentous attachments undisturbed. It is followed by laminectomy, with minimal muscle dissection from the laminae. After decompression, split sections of the spinous process are reconstructed and repositioned with sutures [4].

This approach preserves posterior lumbar supporting structures for spinal stability and prevents atrophy of the paraspinal muscle, allowing for better exposure of intraspinal nerve tissues and adequate decompression of the spinal canal. The indications for trumpet laminectomy microdecompression are similar to those of standard lumbar decompression. Patients with degenerative lumbar canal stenosis and predominant leg pain who have failed in conservative measures are good surgical candidates, regardless of the number of segments [5].

The purpose of this study is to describe the trumpet laminectomy microdecompression technique and intraoperative data, followed by a discussion of its advantages and disadvantages.

\section{Technical Notes}

We performed a review of all patients who underwent trumpet laminectomy microdecompression by neurosurgeons in the Department of Neurosurgery, Aichi Medical University Hospital (Nagakute, Aichi, Japan) between January 2012 to December 2012.

\section{Surgical technique}

All patients underwent surgery after induction of general endotracheal anesthesia. They were placed in the prone position on the hall frame, with lumbar lordosis minimized by flexing the hip joints. An operating microscope was used in all cases, and surgery was performed in a standardized manner. The interspinous space lumbar level to be approached was confirmed using lateral X-ray image and marked with indigo-dye injection into the interspinous space. The back of the patient was washed with aseptic and antiseptic procedures and then draped in a standard surgical fashion.

A great attention was paid to confirm the anatomical orientation (Fig. 1). A midline skin incision of $30 \mathrm{~mm}$ for one-level trumpet laminectomy or $50 \mathrm{~mm}$ for two-level trumpet laminectomy was made. The spinous process was exposed $20 \mathrm{~mm}$ wide, centered at the interspinous level to be decompressed. While preserving the supraspinous ligament and the interspinous ligament, the paravertebral muscle and the capsular facet were left completely intact.

A sharp midline (longitudinal) incision was done with a 2-mm high-speed drill or Williams microsurgical saw, preserving the ligamentous attachment to the rostral part of the spinous process (Fig. 2). Then the tips of the spinous process were opened using a Cobb dissector, and the base of the spinous process was cut using a $2-\mathrm{mm}$ high-speed drill or an L-shape Williams microsurgical saw.

The base of the spinous process was sutured with 0-PDS sutures, which were placed in cranial part of the base spinous process (Fig. 3). The operative field was exposed using two right-angled Koyama self-retaining retractors. The same procedure can be repeated for multiple-level decompression as necessary. Operative field was opened using high-speed drills and Kerrison punches from $1 / 3$ to $1 / 2$ of the caudal part of the involved laminae. The width of trumpet laminectomy is usually $20 \mathrm{~mm}$. Resection of $1 / 4$ medial of the facet joint was done for patients with a hypertrophic facet joint; resection of the intracanal granulation tissue was done for patients with hypertrophic tissue; flavectomy was done for patients with hypertrophic yellow ligament; and herniectomy was done for patients with disc herniation.

Bilateral decompression was done as usual, after meticulous hemostasis by bone wax and Aviten (Fig. 4). After decompressing the neural tissues, the split spinous process is reconstructed and repositioned. This is done by first making two parallel sutures from the caudal to cranial end of the split spinous process, and then pulling together both sutures tightly at the tip and base of the spinous process, using 0-PDS sutures. After reconstruction of the spinous process described above, we performed suturing of the supraspinous ligament with a nonabsorbable suture material. The wound was closed anatomically 
by absorbable suture and stapler layer-by-layer, and an epidural-subcutaneous drain was placed.

Using this technique, adequate decompression can be performed even in patients with narrow and steep facet joints and/or severe canal stenosis, in whom sufficient decompression with facet preservation is difficult to achieve by conventional fenestration (Fig. 5).

\section{Postoperative care}

Patients were observed in the postoperative recovery room and sent to inpatient ward. Patients and family members were informed of the intraoperative findings, wound care and further follow-up. Once the patient was able to walk and had normal vegetative functions, the patient was discharged from the hospital.

\section{Results}

Sixty-two consecutive patients with lumbar disc herniation, facet joint hypertrophy, yellow ligament hypertrophy or intracanal granulation tissue were selected for this
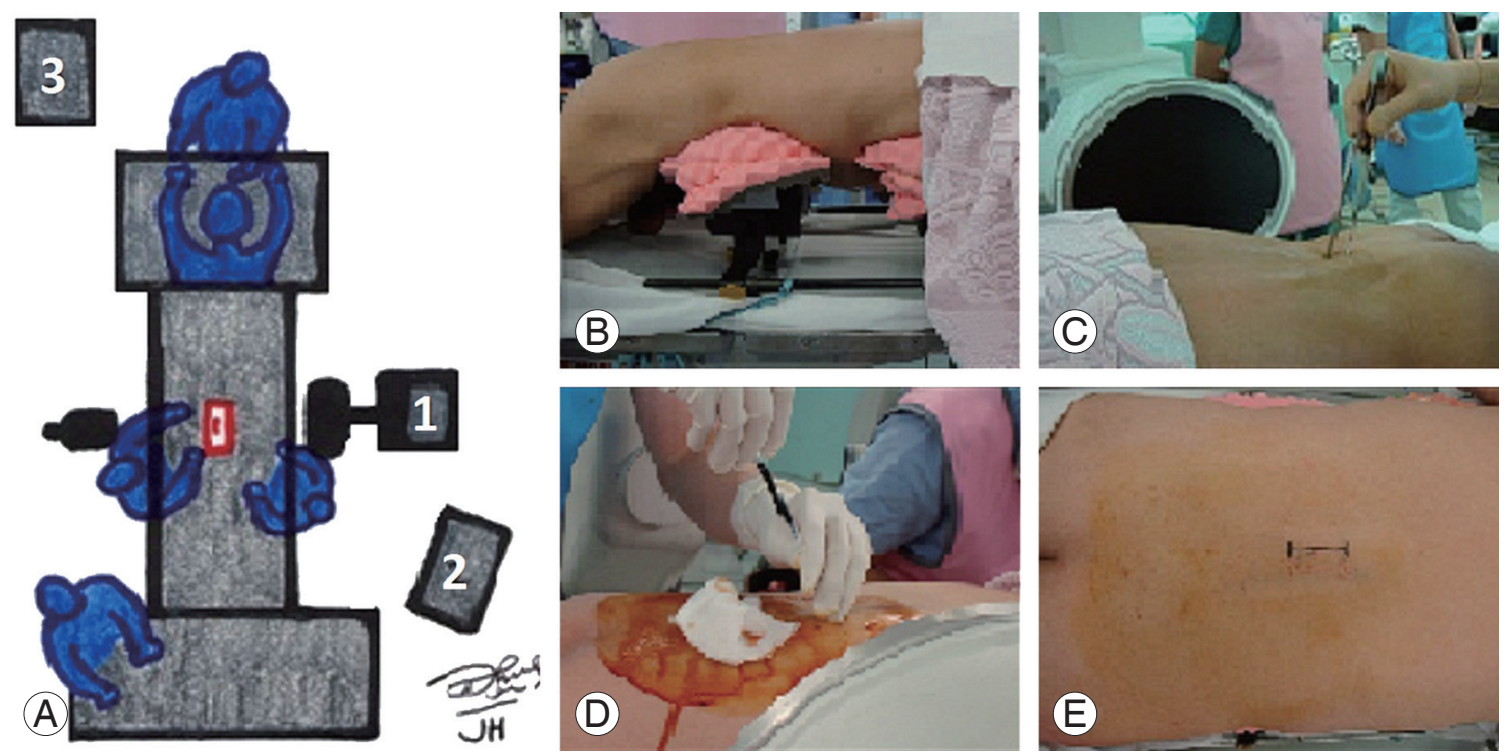

Fig. 1. Patient in the operating room and surgical preparation. (A) Schematic illustration of the operating room set up: (1) X-ray device, (2) X-ray monitor, (3) anestesiologist monitor. (B) Prone position of the patient on the Hall frame. (C) The interspinous space lumbar level was confirmed using lateral X-ray image. (D) Marked with indigo-dye injection into the interspinous space of responsible level. (E) $30 \mathrm{~mm}$ midline skin marked to incision.
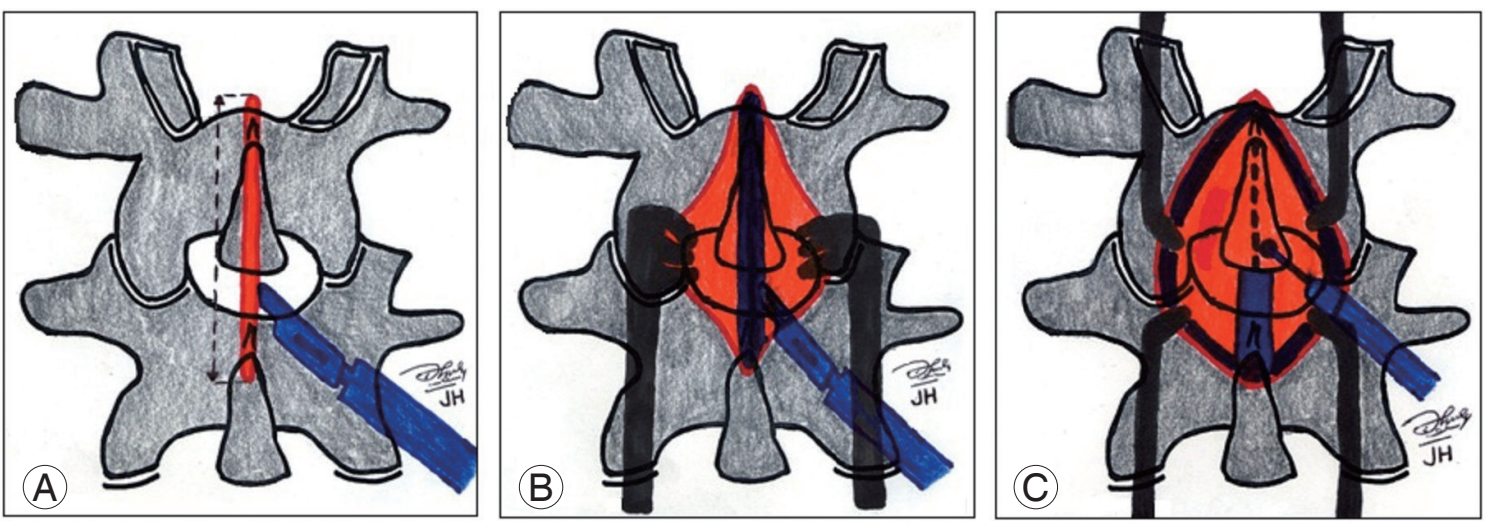

Fig. 2. Schematic illustration of surgical technique for trumpet laminectomy microdecompression. (A) Margin of skin incision on midline of the spinous process. (B) The supraspinous ligament incision on midline of the spinous process and after subcutis retracted with retractor. (C) Midline cutting of the spinous process with 2-mm high-speed drills or Williams microsurgical saw. 
retrospective study. The patients had an average age of 65.8-year-old (range, 13-83-year-old) and included 32 males and 30 females (Table 1).

Commonly the patient complained of low back pain, dysesthesia and severe pain on both legs with intermittent claudication. Of the patients, $17.7 \%$ had a history of previous spine surgery at a different level in another hospital. Levels operated on included Th11-12 (1.6\%), L1-2 (1.6\%), L2-3 (22.6\%), L3-4 (45.2\%), L4-5 (74.2\%), and L5-S1 (9.7\%) (Table 1).

A trumpet-type fenestration was done using high- speed drills, curretts and Kerrison punches. Diseases treated with trumpet-type fenestration were: $62.9 \%$ facet joint hypertrophy; $11.3 \%$ with intracanal granulation tissue; $79.1 \%$ with hypertrophy of the yellow ligament; and $64.5 \%$ with disc herniation (Table 1 ).

The average duration of the surgical procedure was 68.9 minutes (range, 62-94 minutes); and the average intraoperative blood loss was $47.4 \mathrm{~mL}$ (range, $23-98 \mathrm{~mL}$ ). In this study, intraoperative complications were found at $3.2 \%$ with dural damage, but without cerebrospinal leakage after three months of postoperative follow-up (Table 1).
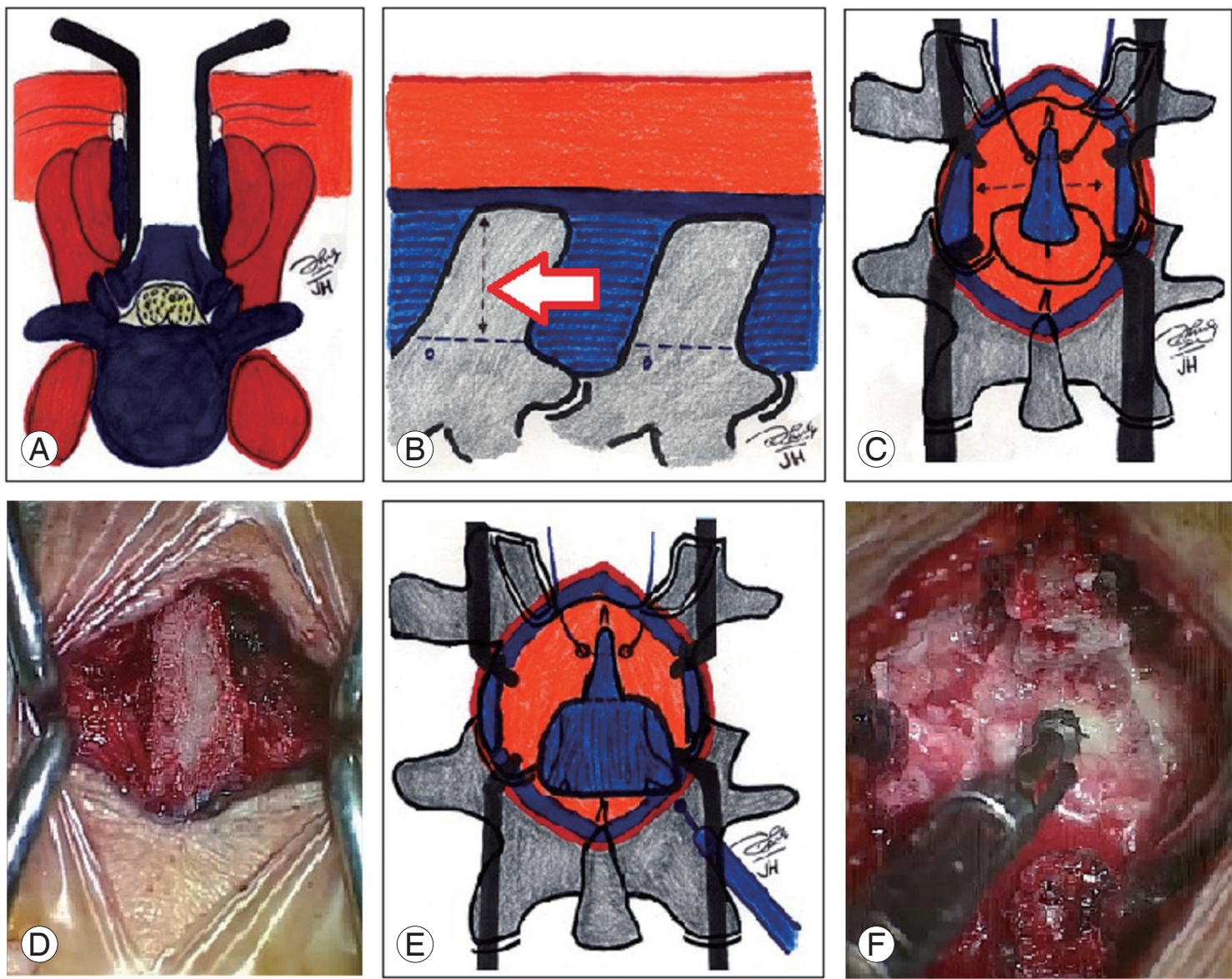

Fig. 3. Schematic illustration and intraoperative microscopic view of surgical technique for trumpet laminectomy microdecompression. (A) Schematic illustration (axial plane) of the spinous process and the supraspinous ligament. After splitting longitudinally in midline with Koyama self-retaining retractors; divided at its base from the lumbar posterior arch, leaving the bilateral paraspinal muscles attached to lateral aspect of the split spinous process. The full exposure of the laminae is now obtained by retracting the split spinous process laterally, together with its attached paraspinal muscle. (B) Schematic illustration (sagittal plane) of the spinous process, the supraspinous ligament and the interspinous ligament. Red arrow is 20-22 mm of high-cutting the spinous process from its base. (C) Schematic illustration of the base spinous process. 0-PDS sutures were placed in cranial part of the base spinous process. (D) Intraoperative microscope view of the base spinous process, removed partially by high speed drills. (E) Schematic illustration of disection and drilling caudal part of the responsible laminae. Performing laminectomy $20 \mathrm{~mm}$ wide by high-speed drills and Kerrison punches. (F) Intraoperative microscope view of drilling caudal part of the responsible laminae. A dome-like expansion of the upper adjacent laminae is performed; microdecompression with trumpet type fenestration. 


\section{Discussion}

In conventional laminectomy for lumbar canal stenosis, the bilateral paraspinal muscles are dissected and detached extensively from the spinous process and the laminae. An advantage of conventional laminectomy is that it provides good visibility and adequate working space by removing posterior elements, including the spinous process, the supraspinous ligament and the interspinous ligament. It enables sufficient decompression of the neural structures by unroofing the spinal canal [3-6]. The disadvantages of conventional laminectomy include the resection of osteoligamentous structure, which sometimes causes secondary spinal instability and trunk extensor weakness. Other minimally invasive techniques could attempt to preserve osseus and ligamentous structures, but these retained midline structures limit visualization, access to the nerve tissue and access to decompression in the lateral recesses. It also still requires stripping of the paraspinal musculature. The potential risk for neural in-
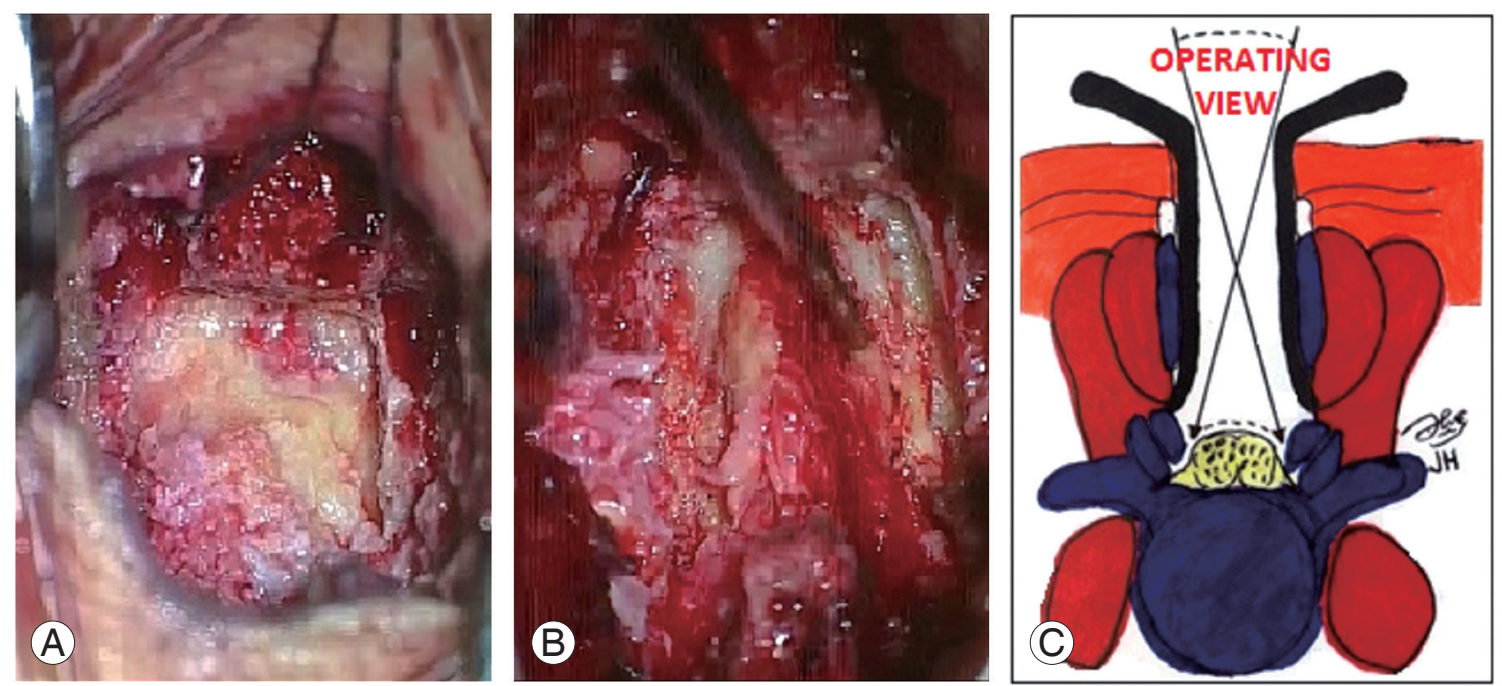

Fig. 4. Intraoperative microscopic view and schematic illustration (axial plane) of trumpet laminectomy microdecompression. (A) Intraoperative microscope view. Bilateral decompression or a dome laminectomy 20-mm wide by high-speed drills and Kerrison punches. (B) The yellow ligament was exposed. (C) Schematic illustration (axial plane) of trumpet type fenestration with large operating view to the lateral recesses.
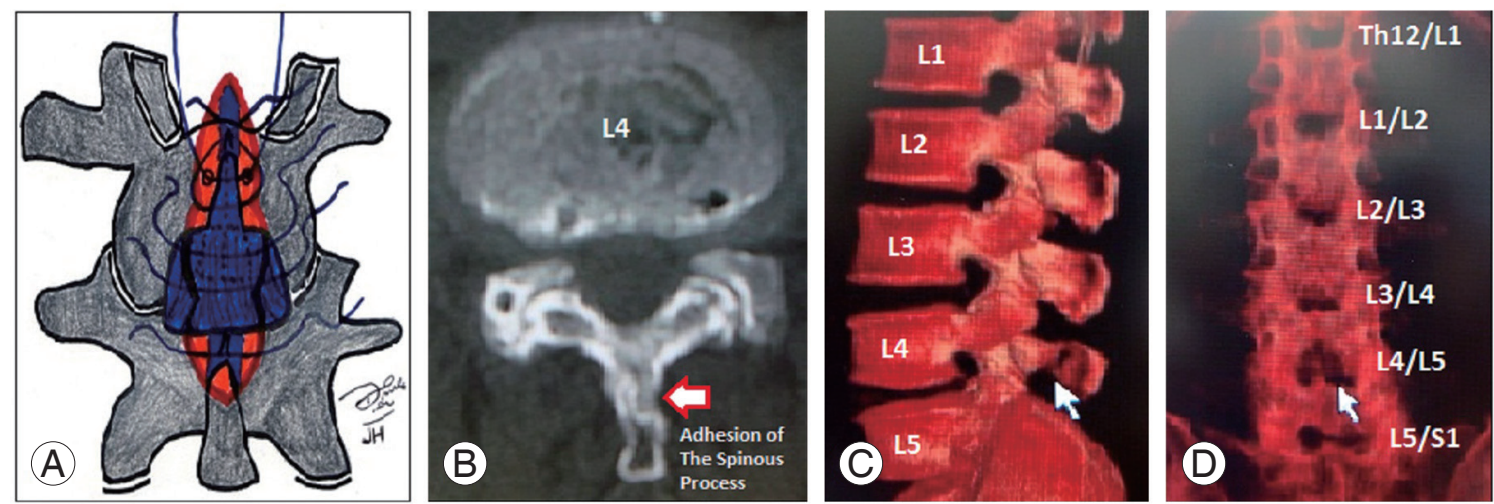

Fig. 5. Schematic illustration and computed tomography (CT) scan, three-months postoperative of trumpet laminectomy microdecompression. (A) Schematic illustration of reconstruction and reposition of the spinous process by sutures. (B) Axial plane of CT scan, three-months postoperative of trumpet laminectomy microdecompression. Red arrow is adhesion of the split spinous process of $L 4$ level. (C) Sagital plane of three-dimensional CT (3D-CT) scan, three-months postoperative of trumpet laminectomy microdecompression. White arrow is the spinous process of $L 4$ level; anatomical reconstruction or reposition and adhesion are clearly visible. (D) Coronal posterior plane of 3D-CT scan, three-months postoperative of trumpet laminectomy microdecompression. White arrow is space of the interlaminar L4/L5 level after trumpet laminectomy microdecompression. 
Table 1. The characteristics of 62 trumpet laminectomy microdecompression patients

\begin{tabular}{|c|c|}
\hline Characteristic & $\begin{array}{l}\text { No. of patients } \\
(n=62)\end{array}$ \\
\hline Age (yr) & 13-83 (mean 65.8) \\
\hline Male:female ratio & $32: 30: 00$ \\
\hline Preoperative symptom & $62(100.0)$ \\
\hline Low back pain & $44(70.9)$ \\
\hline Severe pain on leg & $44(70.9)$ \\
\hline Disestesia & $32(51.6)$ \\
\hline Intermittent claudication & $11(17.7)$ \\
\hline Motor weakness & $2(3.2)$ \\
\hline Difficult in urinary and defecation & $11(17.7)$ \\
\hline \multicolumn{2}{|l|}{ History with previous spine surgery } \\
\hline Level & $1(1.6)$ \\
\hline Th11-12 & $1(1.6)$ \\
\hline $\mathrm{L} 1-2$ & $14(22.6)$ \\
\hline L2-3 & $28(45.2)$ \\
\hline L3-4 & $46(74.2)$ \\
\hline$\lfloor 4-5$ & $6(9.7)$ \\
\hline \multicolumn{2}{|l|}{ L5-S1 } \\
\hline \multicolumn{2}{|l|}{ Intraoperative findings } \\
\hline Yellow ligament hypertrophy & $49(79.1)$ \\
\hline Disc herniation & $40(64.5)$ \\
\hline Facet hypertrophy & $39(62.9)$ \\
\hline Intracanal granulation tissue & $7(11.3)$ \\
\hline Duration of surgical procedures (min) & 62-94 (mean 68.9) \\
\hline Intraoperative blood loss (mL) & 23-98 (mean 47.4) \\
\hline \multicolumn{2}{|l|}{ Intraoperative problems } \\
\hline Dural damage & $2(3.2)$ \\
\hline \multicolumn{2}{|l|}{ Postoperative complications } \\
\hline Pain & $14(22.58)$ \\
\hline Cerebrospinal fluid leakage & $0(0.0)$ \\
\hline Wound dehiscence & $0(0.0)$ \\
\hline
\end{tabular}

jury in a small working space is also a problem, especially in patients with severe central stenosis or narrow and steep facet joints $[3,4,6,7]$.

The success rate of the conventional laminectomy procedure is only $64 \%$. This procedure creates significant intraoperative blood loss, and has frequent surgical failures attributed to local tissue trauma, postoperative incisional pain, prolonged recovery time and possibly failed back-surgery syndrome. The complications caused by iatrogenic back muscle injury is unavoidable in patients undergoing surgery to the lumbar spine [3-7].

Various authors have proposed more tailored and minimally invasive alternative techniques, in particular bilateral and unilateral laminotomy for bilateral decompression, with a reported success rate of $60 \%-80 \%$. Bilateral and unilateral laminotomy prove advantageous for patients, with reduced postoperative pain, no additional fusion surgery and improved health-related quality-oflife. Both of these procedures are superior to conventional laminectomy, but still have several disadvantages such as limited access to the nerve tissue, narrow view of operation for contralateral decompression, residual canal stenosis, inadequate decompression and more common complications such as dural damage or cerebrospinal fluid leakage $[6,8]$.

The trumpet laminectomy microdecompression could minimize the paraspinal muscle damage, and has an advantage in that it preserves the lower levels of the paraspinal muscle from atrophy. According to Mayer et al. [9] and Weiner et al. [10,11], lumbar microdecompression via the spinous process osteotomy avoids stripping of the unilateral paraspinal muscle and yields good outcomes. Shiraishi [12] and Watanabe et al. [7] reported that splitting of the spinous process in laminectomy requires minimal muscle dissection, which are shielded from operative damage, and produces good results.

The trumpet laminectomy microdecompression, which preserves the vitality of the supraspinous or interspinous ligaments and the paraspinal muscles, has an advantage where it can decrease pain intensity and accelerate early patient mobilization, thus reducing the risk of venous thrombosis, atelectasis, urinary tract infection and pneumonia. Jayarao and Chin [4] has reported that the transspinous process approach had a better outcome for pain intensity than conventional laminectomy, with statistical significance.

Conventional laminectomy can cause muscle devitalization and denervation, which may lead to increased oxidative stress and the release of inflamatory prostagnoids and cytokines. This in turn leads to inflammatory cell infiltration and the release of chemical pain mediators. Although no biochemical markers are drawn, the transspinous process approach avoids manipulation of the paraspinal muscle and eliminates the need for its retraction. It thereby likely substantially minimizes the release 
of the chemical mediators and consequently reducing postoperative pain [4].

The trumpet laminectomy microdecompression does not remove the midline osteoligamentous structure. Only $1 / 3$ to $1 / 2$ caudal part of the responsible laminae is drilled away after splitting of the spinous process without manipulation of any musculatures. It has an advantage of preserving spinal stability, while still allowing for better exposure of intraspinal nerve tissues and adequate decompression of the spinal canal. Cho et al. [13] and Abumi et al. [14] reported data from experimental studies, which demonstrated that partial removal of the interspinous ligament or subperiosteal layer of the spinous process is not related to spinal instability.

Matsudaira et al. [15] with modified fenestration and restorative spinoplasty study reported that fenestration with transspinous process is applicable for any type of narrow facet, as confirmed by three-months postoperative threedimensional computed tomography scan follow-up. In the trumpet laminectomy microdecompression technique, it provided the same visibility as conventional laminectomy until the lateral recesses were exposed and intraoperative or postoperative complications were minimal.

A key characteristic of the trumpet laminectomy microdecompression is an anatomical reconstruction or repositioning postoperatively. It rejoins the split spinous process, which provides continuity with the vertebral arch and ensures three-months postoperative spinal stabilization.

The following complications can occur in any lumbar canal stenosis decompression, but are more common in microsurgical approaches. The most common complication is inadvertent dural tear. Where possible, a watertight repair should be performed, but the narrow space for sutures presents difficulties. In this study, there were $3.2 \%$ of patients with intraoperative dural damage, which were overcome by sutures and administration of intraoperative fibrin glue. These patients' results during the three-months postoperative follow-up are without cerebrospinal leakage.

Other potential complications include wound infection, wrong level of surgery, epidural hematome and neurological injury. Wrong level of surgery and neurologic injury can be prevented by careful attention to the anatomic location or landmark of the pathology, by X-ray confirmation and identification of the neurologic structures at all times during the procedure. Little epidural bleed- ing is encountered during lumbar canal decompression; however, epidural venous bleeding is common in lateral side. When encountered, it is easly tamponaded with a small strip of gelfoam or a cottonoid and placed epiduralsubcutaneus drain.

The thin spinous process and osteoporotic present real challenges for operators in performing trumpet laminectomy microdecompression. However, this study did not measure the thickness of the spinous process. The authors suggest performing this technique by splitting the spinous process in the midline, using a thin saw, such as William microsurgical saw or small drill with a maximum size of $<2 \mathrm{~mm}$. It is important not to leverage the spinous process while releasing the basis of the spinous process bone from the laminae. The spinous process fracture while splitting was not found in this study in osteoporosis cases. These problems can be avoided if the technique is conducted carefully at all stages.

The trumpet laminectomy microdecompression has many advantages: no significant muscle or tissue damage; minimal postoperative pain; maintaining spinal stability; earlier mobilization; good functional neurological recovery; and improved quality-of-life. The trumpet laminectomy microdecompression can be performed for lumbar canal stenosis patients of all ages and at all levels of spinal canal stenosis without spondilolistesis complication. The operation has a short duration of surgical procedures and minimal intraoperative blood loss. The trumpet laminectomy microdecompression technique is still evolving and requires some study to prove its advantages and disadvantages in the technique.

\section{Conflict of Interest}

No potential conflict of interest relevant to this article was reported.

\section{Acknowledgments}

The authors would like to thank Rully H. Dahlan and Agung B. Sutiono from Department of Neurosurgery, Faculty of Medicine, Universitas Padjadjaran-Dr. Hasan Sadikin Hospital, Bandung, for fruitful of discussions.

\section{References}

1. Caspar W, Papavero L, Sayler MK, Harkey HL. Pre- 
cise and limited decompression for lumbar spinal stenosis. Acta Neurochir (Wien) 1994;131:130-6.

2. Guiot BH, Khoo LT, Fessler RG. A minimally invasive technique for decompression of the lumbar spine. Spine (Phila Pa 1976) 2002;27:432-8.

3. Young JP, Young PH. Microscopic approach to the posterior lumbar spine for decomprssion. In: Bridwell KH, Dewald RL, editors. The textbook of spinal surgery. Philadelphia: Lippincott; 2012. p.101-9.

4. Jayarao M, Chin LS. Results after lumbar decompression with and without discectomy: comparison of the transspinous and conventional approaches. Neurosurgery 2010;66:152-60.

5. Ehni BL, Benzel EC, Biscup RS. Lumbar discectomy. In: Benzel EC, editor. Spine surgery: techniques, complication avoidance, and management. Philadelphia: Elsevier-Churchill Livingstone; 2005. p.601-18.

6. Thome $\mathrm{C}$, Zevgaridis $\mathrm{D}$, Leheta $\mathrm{O}$, et al. Outcome after less-invasive decompression of lumbar spinal stenosis: a randomized comparison of unilateral laminotomy, bilateral laminotomy, and laminectomy. J Neurosurg Spine 2005;3:129-41.

7. Watanabe K, Matsumoto M, Ikegami T, et al. Reduced postoperative wound pain after lumbar spinous process-splitting laminectomy for lumbar canal stenosis: a randomized controlled study. J Neurosurg Spine 2011;14:51-8.

8. Oertel MF, Ryang YM, Korinth MC, Gilsbach JM, Rohde V. Long-term results of microsurgical treat- ment of lumbar spinal stenosis by unilateral laminotomy for bilateral decompression. Neurosurgery 2006;59:1264-9.

9. Mayer TG, Vanharanta H, Gatchel RJ, et al. Comparison of CT scan muscle measurements and isokinetic trunk strength in postoperative patients. Spine (Phila Pa 1976) 1989;14:33-6.

10. Weiner BK, Fraser RD, Peterson M. Spinous process osteotomies to facilitate lumbar decompressive surgery. Spine (Phila Pa 1976) 1999;24:62-6.

11. Weiner BK, Walker M, Brower RS, McCulloch JA. Microdecompression for lumbar spinal canal stenosis. Spine (Phila Pa 1976) 1999;24:2268-72.

12. Shiraishi T. Skip laminectomy--a new treatment for cervical spondylotic myelopathy, preserving bilateral muscular attachments to the spinous processes: a preliminary report. Spine J 2002;2:108-15.

13. Cho DY, Lin HL, Lee WY, Lee HC. Split-spinous process laminotomy and discectomy for degenerative lumbar spinal stenosis: a preliminary report. J Neurosurg Spine 2007;6:229-39.

14. Abumi K, Panjabi MM, Kramer KM, Duranceau J, Oxland T, Crisco JJ. Biomechanical evaluation of lumbar spinal stability after graded facetectomies. Spine (Phila Pa 1976) 1990;15:1142-7.

15. Matsudaira K, Yamazaki T, Seichi A, et al. Modified fenestration with restorative spinoplasty for lumbar spinal stenosis. J Neurosurg Spine 2009;10:587-94. 